\title{
Tunneling times in a taut string
}

\author{
Diosdado Villegas* \\ Instituto de Física, Benemérita Universidad Autónoma de Puebla, Apartado Postal J-48, CP 7250 Puebla, México
}

Francisco A. Horta-Rangel, Tamé González, and Israel Quirós

Departamento de Ingeniería Civil, División de Ingeniería, Universidad de Guanajuato, Gto., México

R. Pérez-Álvarez

Centro de Investigación en Ciencias-(IICBA), Universidad Autónoma del Estado de Morelos, Ave. Universidad 1001, CP 62209, Cuernavacas, Morelos, México

Fernando de León-Pérez ${ }^{\dagger}$

Centro Universitario de la Defensa de Zaragoza,

Ctra. de Huesca s/n, E-50090, Zaragoza, Spain

(Dated: March 15, 2020)

The mathematical analogy of classical and matter waves can help to teach the elusive subject of tunneling times in undergraduate physics courses. The tunneling of mechanical energy through a taut string is revisited in this paper in order to study tunneling times for this classical system. General properties of the group delay, the dwell time and the interference time are described. Moreover, we explain how to build string arrays with piecewise constitutive parameters that behave like quantum mechanical heterostructures with alternating well and barrier layers. The paradoxical Hartman effect is also analyzed.

PACS numbers: 68.65.Cd, 63.20.Dj, 63.22.1m 


\section{INTRODUCTION}

Analogies between quantum phenomena described by the time-independent Schrödinger equation and the classical world can be drawn provided that the equations of motion of classical systems can be written as Helmholtz equations. Limiting ourselves to elastic waves and acoustics, we can mention among such analogies the classical Anderson localization in 1D acoustical system, ${ }^{1,2}$ acoustic Bloch oscillations, ${ }^{3}$ acoustic Wannier-Stark ladders, ${ }^{4-6}$ and doorway states in quasi-1D elastic systems. ${ }^{7}$ The physics of quantum tunneling has also been extrapolated to modes of vibration of elastic structures that account for interacting particles in crystals. ${ }^{8-10}$ Such vibrational modes can be described as classical elastic waves.

The group delay or phase time $\tau_{g}$ is one of the important quantities related to the dynamic aspect of the tunneling. In quantum mechanics, it is defined as the derivative of the phase shift of a wavepacket with respect to energy. It is surprising to find that $\tau_{g}$ for a particle tunneling through a rectangular barrier is independent of the barrier thickness provided the barrier is opaque. This phenomenon is often referred to as the Hartman effect (HE). ${ }^{11}$ Notice that the particle travels with a group velocity $v_{g}=L / \tau_{g}$. If phase time becomes a constant while the barrier length increases, the group velocity will exceed the speed of light in vacuum.

The HE has been verified by a series of experiments measuring the delay time between the appearance of an electromagnetic pulse peak at the two sides of a barrier. ${ }^{12-18}$ The existence of superluminal group velocities in tunneling has been attributed to a reshaping phenomenon. ${ }^{14}$ The accepted explanation for this counterintuitive behavior is that the barrier transmits the early parts of the incident pulse and rejects the later parts. This argument is based on the analogy between electromagnetic tunneling of evanescent waves and quantum-mechanical tunneling. Winful ${ }^{19}$ proposed the alternative argument that the measured delays are not propagation delays and, therefore, the saturation of the group delay time does not imply superluminal and unlimited velocity. It is worth to notice that the isomorphism between quantum tunneling and tunneling of vibrational modes has been exploited to interpret the paradoxical HE for acoustic waves. ${ }^{20-22}$

In this paper, we revisit the canonical system for elastic waves, a taut string, which is typically discussed in undergraduate physics courses. ${ }^{23,24}$ Despite its simplicity, it can greatly assist engineer and physics students in understanding the vibration of continuous systems and wave phenomena in layered systems. Recent experimental ${ }^{25-28}$ and theoretical ${ }^{29-32}$ studies confirm the interest on this system.

Our attention is focused on the tunneling of mechanical energy through a taut string. The main goal of this paper is to show that tunneling times, a controversial subject of quantum mechanics, ${ }^{19}$ can be naturally defined and analyzed in simple terms with help of this basic model. We find here that group delays and dwell times in a taut string are equivalent to those times computed for other kind of waves. In particular, we shall discuss the HE.

The systems considered in this paper are schematically represented in Fig. 1. Fig. 1 (a) shows a taut string of mass density $\rho_{s_{2}}$, phase velocity $v_{s_{2}}$ and thickness $L$ sandwiched between two semi-infinite strings. We assume that a transverse wave, excited in the infinite string I, impinges at the boundary with the finite string II. Part of the mechanical energy is reflected back to medium I and the remaining energy is transmitted to the infinite string III.

The strings in Fig. 1 (a) are characterized by a linear dispersion relation. Thus, transverse waves traveling trough

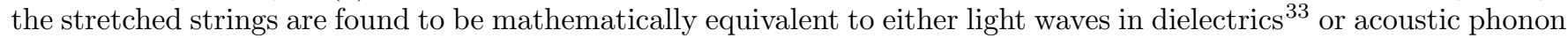
waves. ${ }^{34}$ Dispersive waves can be excited by placing the string on an elastic foundation, as in Fig. 1 (b), which is modelled as a transverse elastic force that provides a quadratic dispersion relation. ${ }^{23,24}$ Such dispersive waves are equivalent to either matter waves or optical vibrational modes in solids. ${ }^{8}$ We shall also compute the tunneling times of a periodic array of strings with piecewise constitutive parameters. This idealized model is depicted in Fig. 1(c).

The paper is organized as follows. Section II studies the tunneling through a single taut string. The general properties of its wave equation and the corresponding continuity equation are first described in this section. Next, the different tunneling times are defined. Such quantities are used to explain the Hartman effect. Section III extends the results to an array of strings. Several mathematical derivations and lengthy calculations are moved to the appendixes for the sake of clarity. The main conclusions are given at the end of the paper.

\section{TUNNELING THROUGH A SINGLE STRING}

The general approach to the tunneling problem is applied to the single taut strings shown in Figs. 1 (a) and (b). Their relevant tunneling times are defined and characterized in this section. Our didactic approach is organized as follows: (i) We start with the equation of motion of the classical systems. (ii) A continuity equation for the energy density is derived from the equation of motion. (iii) The group delays that characterize the tunneling time are calculated using the method of stationary phase. (iv) The dwell time is written in terms of the mechanical energy accumulated in the system. (v) A mathematical relation between group delay and dwell time is derived from the Helmholtz equation. 


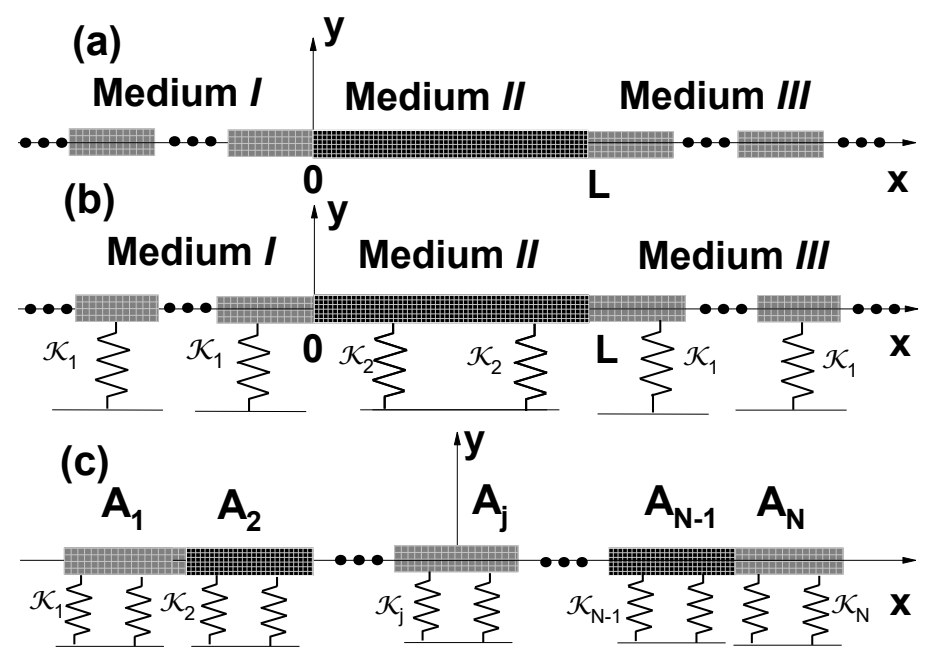

FIG. 1. (a) A taut string of mass density $\rho_{s_{2}}$ and length $L$ is sandwiched between two identical semi-infinite strings. (b) The same string on an elastic base. (c) A periodic array of strings on elastic base. The substrate and detector vibrational properties are assumed to be the same in all numerical calculations.

\section{A. Wave equation}

The wave equation for the transverse waves of an ideal string,

$$
\frac{\partial^{2} y}{\partial t^{2}}=v_{s}^{2} \frac{\partial^{2} y}{\partial x^{2}}
$$

is obtained by applying second Newton law to an infinitesimal portion of the string. ${ }^{24,30}$ We have assumed a linearly elastic string without flexural rigidity, so the only restoring force acting on string elements is a tensile force $T$, which is tangential to the local string direction. Under such conditions, the string oscillates along the vertical direction. The displacement from equilibrium at any time of this transverse wave can be specified by the coordinate $y(x, t)$. The wave propagates with constant speed $v_{s}=\sqrt{T / \rho_{s}}$ in a non-dispersive medium, where $\rho_{s}$ is the linear mass density of the string. The relationships between wave number $k$, angular frequency $\omega$, frequency $f$ and wavelength $\lambda$ are $k=2 \pi / \lambda=\omega / v_{s}$, where $\omega=2 \pi f$ and $v_{s}=\lambda f$. Longitudinal displacements of string points caused by nonlinear effects $^{31,32}$ will be neglected here for this subject largely exceeds the didactic goals of this paper.

When the string is supported by an elastic foundation with elastic modulus $\mathcal{K}$, the vertical elastic force per unit length $\mathcal{F}_{e}=-\mathcal{K} y(x, t)$ must be added to the wave equation, ${ }^{23,24}$

$$
\frac{\partial^{2} y}{\partial t^{2}}=-\omega_{c}^{2} y+v_{s}^{2} \frac{\partial^{2} y}{\partial x^{2}}
$$

where $\omega_{c}=\sqrt{\mathcal{K} / \rho_{s}}=v_{s} \sqrt{\mathcal{K} / T}$ is the cutoff frequency.

We consider that a one-dimensional wave with unit amplitude, $e^{i k_{1} x}$, is incident from medium I. Harmonic solutions are assumed in what follows and the factor $\exp (-i \omega t)$ is omitted for the sake of simplicity. Both Eqs. (1) and (2) have the same solution that, for the piecewise material shown in Figs. 1(a) and (b), can be written as

$$
y(x)= \begin{cases}e^{i k_{1} x}+r e^{-i k_{1} x}, & x<0, \\ A e^{i k_{2} x}+B e^{-i k_{2} x}, & 0 \leq x \leq L, \\ t e^{i k_{1}(x-L)}, & x>L .\end{cases}
$$

The unknown constants $t, r, A$ and $B$ can be determined from the boundary conditions: continuity at $x=0$ and $x=L$ of both the displacement field $y(x)$ and the stress field

$$
\sigma=T \frac{\partial y}{\partial x}
$$


In fact, we use the continuity of $\partial y / \partial x$ for $T=\rho_{s} v_{s}^{2}$ is constant. The quantities

$$
\begin{aligned}
t & =\frac{1}{\cos \left(k_{2} L\right)-i \frac{\tilde{\xi}_{m}}{2} \sin \left(k_{2} L\right)}, \\
r & =-\frac{i}{2} \tilde{\xi}_{n} \sin \left(k_{2} L\right) t
\end{aligned}
$$

are the reflected and transmitted amplitudes, respectively, where $\tilde{\xi}_{m}=Z_{1} / Z_{2}+Z_{2} / Z_{1}, \tilde{\xi}_{n}=Z_{1} / Z_{2}-Z_{2} / Z_{1}$, and $Z_{i}=\rho_{s_{i}} v_{s_{i}}^{2} k_{i}$, with $i=1,2$. These complex quantities can be written as $t=|t| \exp \left(i \alpha_{t}\right)$ and $r=|r| \exp \left(i \alpha_{r}\right)$. The transmission and reflection coefficients are defined as the squared absolute values of $t$ and $r$,

$$
\begin{aligned}
\mathcal{T} & \equiv|t|^{2}=\frac{1}{1+\frac{\tilde{\xi}_{n}^{2}}{4} \sin ^{2}\left(k_{2} L\right)}, \\
\mathcal{R} & \equiv|r|^{2}=\frac{1}{4} \tilde{\xi}_{n}^{2} \sin ^{2}\left(k_{2} L\right) \mathcal{T}
\end{aligned}
$$

which fulfill the identity $\mathcal{T}+\mathcal{R}=1$. Their respective phases,

$$
\begin{aligned}
& \alpha_{t}=\arctan \left(\frac{\tilde{\xi}_{m}}{2} \tan \left(k_{2} L\right)\right), \\
& \alpha_{r}=-\pi / 2+\arctan \left(\frac{\tilde{\xi}_{m}}{2} \tan \left(k_{2} L\right)\right),
\end{aligned}
$$

will be used below for computing the tunneling times. The amplitudes of the field in medium II are equal to

$$
\begin{aligned}
& A=\frac{1}{2}\left(1+\frac{Z_{1}}{Z_{2}}\right) t e^{-i k_{2} L}, \\
& B=\frac{1}{2}\left(1-\frac{Z_{1}}{Z_{2}}\right) t e^{i k_{2} L} .
\end{aligned}
$$

Nonetheless, the above solution yields different wavenumbers for Eqs. (1, 2). The wave of Eq. (1) is not dispersive due to the linear relation between wavenumber and angular frequency,

$$
k_{i}=\frac{2 \pi f}{v_{s_{i}}},
$$

with $i=1,2$. Equivalent linear dispersion relations are found for acoustic waves and for the light traversing lossless dielectric materials. By contrast, the wave becomes dispersive in the presence of an elastic foundation. The dispersion relation that satisfies Eq. (2) is a quadratic function: $f^{2}=\left(v_{s_{i}} / 2 \pi\right)^{2} k_{i}^{2}+f_{c_{i}}^{2}$. Similar dispersion relations are found for electrons and optical phonons. The functional form of the wavenumber,

$$
k_{i}=\frac{2 \pi}{v_{s_{i}}} \sqrt{f^{2}-f_{c_{i}}^{2}},
$$

determines that the wave is at cutoff for the frequency $f_{c_{i}}=\left(v_{s_{i}} / 2 \pi\right) \sqrt{\mathcal{K}_{i} / T}$. The wave can freely propagate along the string with a real $k_{i}$ when the frequency is beyond cutoff, while, below cutoff, the string behaves as a potential barrier for the incident wave because it decays for imaginary values of $k_{i}$. The solution for such non-propagating waves will be written as

$$
y(x)=A e^{-\kappa x}+B e^{\kappa x},
$$

for the central string $(0 \leq x \leq L)$, where $\kappa=-i k_{2}$ is used for the sake of simplicity.

\section{B. Continuity equation}

The continuity equation for the energy density

$$
\frac{\partial \mathcal{H}}{\partial t}+\frac{\partial j}{\partial x}=0
$$


can be derived from the wave equation (see Appendix A). The energy density flux $j$ is

$$
j=-\frac{1}{2}\left(\sigma \frac{\partial y^{*}}{\partial t}+\sigma^{*} \frac{\partial y}{\partial t}\right)
$$

for both Eqs. (1) and (2). One can check that both transmission (5) and reflection (6) coefficients can be computed as $\mathcal{T}=j_{t} / j_{\text {in }}$ and $\mathcal{R}=j_{r} / j_{i n}$, in terms of the transmission, $j_{t}=\rho_{s_{1}} \omega v_{s_{1}}^{2} k_{1}\left|t\left(k_{1}\right)\right|^{2}$, reflection, $j_{r}=\rho_{s_{1}} \omega v_{s_{1}}^{2} k_{1}\left|r\left(k_{1}\right)\right|^{2}$, and incident, $j_{i n}=\rho_{s_{1}} \omega v_{s_{1}}^{2} k_{1}$, fluxes, respectively.

The energy density is given by

$$
\mathcal{H}_{s}=\frac{1}{2} \rho_{s}\left|\frac{\partial y}{\partial t}\right|^{2}+\frac{1}{4}\left(\sigma \frac{\partial y^{*}}{\partial x}+\sigma^{*} \frac{\partial y}{\partial x}\right)
$$

for the standard taut string (Eq. 1) and by

$$
\mathcal{H}_{e f}=\frac{1}{2} \rho_{s}\left|\frac{\partial y}{\partial t}\right|^{2}+\frac{1}{4}\left(\sigma \frac{\partial y^{*}}{\partial x}+\sigma^{*} \frac{\partial y}{\partial x}\right)+\frac{1}{2} \rho_{s} \omega_{c}^{2}|y|^{2}
$$

for the string on the elastic foundation (Eq. 2). In the above equations, the first term represents the kinetic energy density and the second one is the strain energy density. The third term in Eq. (14) is the density of elastic potential energy related to the interaction of the string with its elastic foundation.

The total mechanical energy accumulated in the scattering region $[0, L]$ is equal to

$$
H=\int_{0}^{L} \mathcal{H} d x
$$

It yields

$$
H=\rho_{s_{2}} \omega^{2}\left(|A|^{2}+|B|^{2}\right) L+\frac{\rho_{s_{2}} \omega_{c_{2}}^{2}}{k_{2}}|A||B|\left[\sin \left(2 k_{2} L+\Lambda\right)-\sin (\Lambda)\right],
$$

for propagating waves in a string on the elastic foundation, where we defined $A=|A| e^{i \alpha}, B=|B| e^{i \beta}$, and $\Lambda=\alpha-\beta$. The second term vanishes for a simple taut string (with $\omega_{c_{2}}=0$ ). In turn, $H$ is more involved for non-propagating waves,

$$
H=\frac{\rho_{s_{2}} \omega_{c_{2}}^{2}}{2 \kappa_{2}}\left[|A|^{2}\left(1-e^{-2 \kappa_{2} L}\right)+|B|^{2}\left(1-e^{2 \kappa_{2} L}\right)\right]+2 \rho_{s_{2}} \omega^{2}|A||B| L \cos (\Lambda) .
$$

\section{Tunneling times}

In this section, group delay and dwell time are defined and a mathematical relation is established between these quantities. We follow a general approach based in the stationary phase method, which is valid for an arbitrary onedimensional wave packet. This approach is briefly reviewed here for the sake of completeness. At the end of the section, we will compute explicit expressions for tunneling times of the two particular systems shown in Figs. 1 (a) and (b).

We construct a spatially-localized incident wave packet by superposition of stationary states with different wave numbers as

$$
y_{i n}(x, t)=\int \frac{d k}{2 \pi} \phi\left(k-k_{0}\right) y_{k}(x) e^{-i \omega(k)},
$$

where $\phi\left(k-k_{0}\right)$ is a narrow momentum distribution such as a Gaussian centered around $k_{0}$. We assume that, at $t=0$, the wave packet is centered at $x=0$ and propagates to the right with a group velocity $v_{g}=\frac{\partial w}{\partial k}$. This packet is replaced by reflected and transmitted wave packets. The one reflected at the structure left side $(x<0)$ can be written as

$$
y_{L}(x, t)=\int \frac{d k}{2 \pi} \phi\left(k-k_{0}\right)|r(k)| e^{-i \Phi_{r}(k)},
$$

where $\Phi_{r}(k)=-\alpha_{r}+k x+\omega t$ is the total phase of the reflected wave. Stationary phase method ${ }^{24}$ is the simplest and most common approximation adopted for obtaining the tunneling times of a wave packet in a tunneling process. ${ }^{24}$ 
Assuming that the phase $\Phi_{r}(k)$ varies sufficiently smoothly, the stationary phase method can be successfully applied to describe the movement of the peak of the reflected wave packet. According to this method, the integral (18) can therefore be estimated by finding the value for which the phase has a vanishing derivative. Evaluating the integral in the neighborhood of the $k_{0}$, we obtain

$$
\left.\frac{\partial}{\partial k} \Phi_{r}(k)\right|_{k=k_{0}}=x+\left.\left(-\frac{\partial \alpha_{r}}{\partial k}+\frac{\partial \omega}{\partial k} t\right)\right|_{k=k_{0}}=0 .
$$

The reflected wave-packet peak appears at position $x=0$ with a group delay given by

$$
\tau_{r}=\frac{\partial \alpha_{r}}{\partial \omega}
$$

The transmitted packet at the structure right side $(x>L)$ can be written as

$$
y_{R}(x, t)=\int \frac{d k}{2 \pi} \phi\left(k-k_{0}\right)|t(k)| e^{i \Phi_{t}(k)},
$$

where $\Phi_{t}(k)=\alpha_{t}+k(x-L)-\omega t$ is total phase of the transmitted wave. Applying the stationary phase method results in

$$
\left.\frac{\partial}{\partial k} \Phi_{t}(k)\right|_{k=k_{0}}=x-L+\left.\left(\frac{\partial \alpha_{t}}{\partial k}-\frac{\partial \omega}{\partial k} t\right)\right|_{k=k_{0}}=0 .
$$

In this case, the transmitted wave-packet peak appears at position $x=L$ with the group delay

$$
\tau_{t}=\frac{\partial \alpha_{t}}{\partial \omega}
$$

On the other hand, the dwell time ${ }^{35,37}$ is the time spent by a wave in the scattering region regardless of whether it is ultimately transmitted or reflected. In analogy with matter, electromagnetic, and acoustic waves, we can define the dwell time as

$$
t_{D}=\frac{H}{j_{i n}}
$$

where $H(15)$ is the vibrational energy stored by the scattering system (region $[0, L]$ ) and $j_{\text {in }}$ is incident energy density flux.

Reflection and transmission group delays and the dwell time satisfy the following identity (derived in Appendix B)

$$
t_{D}=\mathcal{T} \tau_{t}+\mathcal{R} \tau_{r}+\tau_{i}
$$

where the interference time

$$
\tau_{i}=\frac{\omega_{c_{1}}^{2}}{\omega v_{s_{1}}^{2} k_{1}^{2}} \sqrt{\mathcal{R}} \sin \left(\alpha_{r}\right)
$$

is only defined for the taut string on the elastic foundation. The interference term comes from the overlap of incident and reflected waves packets in front of the structure. ${ }^{39}$ Alternatively, the interference time is defined as the ratio of the total excess energy to the left of the structure and the incident flux (see Ref. 40 and references therein). We stress that this term vanishes when the elastic base is removed $\left(\omega_{c_{1}}=0\right)$.

Quantities derived up to this point are valid for any one dimensional wave. Particular expressions for the simple systems shown in Fig. 1 are given in what follows. The taut string of Fig. 1(a) is considered first. By substituting Eqs. (7) and (8) into Eqs. (23) and (20), we find that the group delay is proportional to the transmission coefficient,

$$
\tau_{t}=\frac{\tilde{\xi}_{m} \omega L}{2 v_{s_{2}}^{2} k_{2}} \mathcal{T}
$$

In the symmetric configuration considered here (the strings have the same constitutive parameters in media I and III) transmission and reflection times are equal, $\tau_{r}=\tau_{t}$, as pointed out in Refs. 37 and 41. Replacing Eq. (13) into Eq. (15) (or equivalently from Eq. (16) by taking $\omega_{c_{2}}=0$ ), we obtain the mechanical energy

$$
H=\frac{Z_{1}}{Z_{2}} \frac{\rho_{s_{2}} \tilde{\xi}_{m} \omega^{2} L}{2} \mathcal{T}
$$




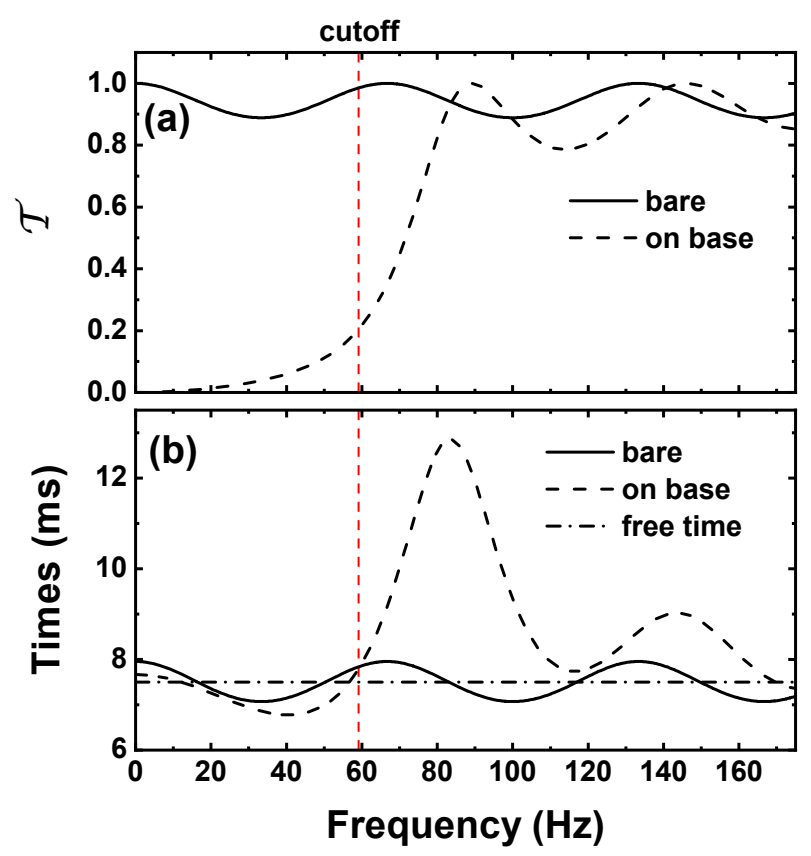

FIG. 2. (a) Transmission coefficient versus frequency for a bare taut string (solid line) and taut string with an elastic base (dashed line). (b) The corresponding group delays are compared with the free time (dash-dotted line). The vertical red-dashed line indicates the position of the cutoff frequency $f_{c_{2}}=59 \mathrm{~Hz}$. The input parameters for the medium $I(I I)$ are $\rho_{s_{1}}=1.0 \mathrm{~g} / \mathrm{cm}$ $\left(\rho_{s_{2}}=0.5 \mathrm{~g} / \mathrm{cm}\right), v_{s_{1}}=40.0 \mathrm{~m} / \mathrm{s}\left(v_{s_{2}}=56.57 \mathrm{~m} / \mathrm{s}\right)$ and $L=42.43 \mathrm{~cm}$. Medium III is identical to medium I. The elastic constants for the elastic foundation in (b) are $\mathcal{K}_{1}=0$ and $\mathcal{K}_{2}=700 \pi^{2} \mathrm{~N} / \mathrm{m}^{2}$. Therefore, only the finite string 2 is coupled to this base.

Notice that for this case $\tau_{t}=t_{D}=H / j_{\text {in }}$ (Eq. 24), in agreement with the identity (25).

Expressions are more involved for the taut string on an elastic foundation, shown in Fig. 1(b). Only propagating states are considered here. For this particular case we find

$$
\begin{aligned}
\tau_{t} & =\mathcal{T} \omega\left[\frac{\tilde{\xi}_{n}}{4}\left(\frac{1}{k_{1}^{2} v_{s_{1}}^{2}}-\frac{1}{k_{2}^{2} v_{s_{2}}^{2}}\right) \sin \left(2 k_{2} L\right)+\frac{\tilde{\xi}_{m} L}{2 k_{2} v_{s_{2}}^{2}}\right], \\
H & =\frac{\rho_{s_{2}}}{2} \frac{Z_{1}}{Z_{2}} \mathcal{T}\left(\omega^{2} \tilde{\xi}_{m} L-\omega_{c_{2}}^{2} \tilde{\xi}_{n} \frac{\sin \left(2 k_{2} L\right)}{2 k_{2}}\right), \\
t_{D} & =\frac{\mathcal{T}}{2 v_{s_{2}}^{2} k_{2}}\left(\omega \tilde{\xi}_{m} L-\frac{\omega_{c_{2}}^{2}}{\omega} \tilde{\xi}_{n} \frac{\sin \left(2 k_{2} L\right)}{2 k_{2}}\right), \\
\tau_{i} & =-\frac{\omega_{c_{1}}^{2} \tilde{\xi}_{n} \mathcal{T}}{4 \omega k_{1}^{2} v_{s_{1}}^{2}} \sin \left(2 k_{2} L\right) .
\end{aligned}
$$

These equations also satisfy the relationship of Eq. (25).

Fig. 2 compares the tunneling through a single taut string (solid lines) and the same string on an elastic base (dashed lines). The transmission coefficient $\mathcal{T}$ is depicted in Fig. 2 (a), while Fig. 2(b) shows the tunneling time in transmission, $\tau_{t}$. Input parameters are given in the figure caption. We assume that only the finite string 2 is coupled to the elastic base. Thus, the interference time (31) vanishes identically as it is proportional to $\omega_{c_{1}}^{2}=\mathcal{K}_{1} / \rho_{s_{1}} \equiv 0$, and group delay (28) and dwell time are (30) identical quantities.

We observed in Fig. 2(a) that the transmission coefficient oscillates as a function of the frequency. The resonant frequencies are obtained by setting $\mathcal{T}=1$ in Eq. (5). This equation is satisfied for $k_{2} L=n \pi$, with $n=0,1,2, \ldots$ The linear dispersion relation of a single taut string of Eq. (9) yields

$$
f_{n}=\frac{n v_{s_{2}}}{2 L} .
$$

The resonant frequencies excited in Fig. 2 (a) are $f_{0}=0 \mathrm{~Hz}, f_{1}=67 \mathrm{~Hz}$ and $f_{2}=133 \mathrm{~Hz}$, for $n=0,1$, and 2, respectively. In contrast, when the taut string is on the elastic foundation, the resulting quadratic dispersion relation, 
Eq. (10), determines that resonances cannot be excited below the cutoff frequency $f_{c}=59 \mathrm{~Hz}$, represented with a vertical red-dashed line in Fig. 2. At the resonant condition $\left(k_{2} L=n \pi\right)$, we have

$$
f_{n}^{e f}=\sqrt{f_{n}^{2}+f_{c_{2}}^{2}}
$$

where $f_{n}$ is the resonant frequency of the bare string (32). Therefore, the resonant frequencies for the string on the elastic foundation are larger than $f_{n}$. The shifted resonances in Fig. 2 are $f_{1}^{e f}=89 \mathrm{~Hz}$ and $f_{1}^{e f}=146 \mathrm{~Hz}$. Also notice that narrower resonances are obtained in the presence of the elastic base.

Fig. 2 (b) shows the group delay for both the bare string $\tau_{t}^{b}$ and the string on the elastic base $\tau_{t}^{e f}$. The group delay oscillates around the free propagation time $\tau_{f}=L / v_{2}$, represented with a horizontal line. $\tau_{f}$ is the time of transit (or free time) of the group pulse through the string. While $\tau_{t}^{b}$ is symmetric with respect to $\tau_{f}$, $\tau_{t}^{e f}$ has asymmetric and broader peaks.

Both $\tau_{t}^{b}(27)$ and $\tau_{t}^{e f}(28)$ are proportional to the transmission coefficient. Therefore, the peaks of the transmission times are at the same spectral positions than the resonances of $\mathcal{T}$. The prefactor of $\tau_{t}^{b}(27)$ has a constant value, equal to $7.97 \mathrm{~ms}$ for the input parameters of Fig. 2. However, the prefactor of $\tau_{t}^{e f}$ (28) is a non-monotonic function of the frequency that produces the asymmetric peaks. In fact, the intensity of the resonant peaks of $\tau_{t}^{e f}$ decays with $f$, being the main peak of $\tau_{t}^{e f}$ higher than the main peak of $\tau_{t}^{b}$. The prefactor of $\tau_{t}^{e f}$ diverges at $f=0$ so its product with $\mathcal{T}$ yields a finite value.

\section{Hartman effect}

The tunneling time becomes independent of the length $L$ of an opaque barrier, except for a very thin or very thick barrier. By opaque barrier we mean that the string is at cutoff $\left(k_{2}=i \kappa\right)$ and $L \kappa \rightarrow \infty$. This seminal result of Hartman, ${ }^{11}$ coined as Hartman effect, suggests the idea of instantaneous propagation that violates our notions of causality. The HE has received much attention and has been already reported for electronic, photonic and phononic waves. The tunneling through a potential barrier ${ }^{42}$ and the quantum tunneling through both single and double barriers in a single graphene layer ${ }^{43}$ are nice examples of the electronic HE. Among the realizations of the photonic HE we can find the tunneling of evanescent electromagnetic waves, ${ }^{39}$ the superluminal propagation in a left-handed medium, ${ }^{44}$ the phenomenon of frustrated total internal reflection of electromagnetic waves ${ }^{45}$ and the tunneling of negative-refractive-index metamaterials. ${ }^{46}$

This paradox was solved by Winful (see Ref 19 and references therein). Winful has shown that the group delay is proportional to the average time in which the electromagnetic energy is stored in the cavity and it is a measure of the cavity lifetime for the electromagnetic waves. He used this proportionality between the group delay and the stored electromagnetic energy to explain the HE paradox: the group delay is not a transit time but a lifetime. This approach eliminates the possibility of superluminal and unbounded group velocities. Arguments similar to those of Winful have been employed to describe the saturation of the group delay for long-wavelength phonons in semiconductor heterostructures. $^{20}$

We illustrate the HE using the taut string in an elastic base of Fig. 1 (b). Non-propagating states are assumed for the string, which behaves like a barrier (for a bare string only propagating states are allowed, so we cannot have a barrier for the incident wave). The wavenumber $k_{2}$ is replaced by $i \kappa$ in the expressions for $\tau_{t}(28), H(29), t_{D}(30)$ and $\tau_{i}(31)$. Taking the limiting values of these quantities for a very large string, i.e. for $L$ approaching $\infty$ when the Hartman effect appears, the resulting expressions become independent of the barrier length:

$$
\begin{aligned}
\tau_{t_{l}} & =\frac{2 \omega}{\hat{\xi}_{m}}\left(\frac{1}{k_{1}^{2} v_{s_{1}}^{2}}+\frac{1}{\kappa^{2} v_{s_{2}}^{2}}\right), \\
H_{l} & =\frac{2 \omega_{c_{2}}^{2} Z_{1}}{\kappa^{2} v_{s_{2}}^{2} \hat{\xi}_{m}}, \\
t_{D_{l}} & =\frac{2 \omega_{c_{2}}^{2}}{\omega \kappa^{2} v_{s_{2}}^{2} \hat{\xi}_{m}}, \\
\tau_{i_{l}} & =-\frac{2 \omega_{c_{1}}^{2}}{\omega k_{1}^{2} v_{s_{1}}^{2} \hat{\xi}_{m}},
\end{aligned}
$$

where the following quantities are redefined to $\hat{\xi}_{m}=Z_{1} / Z_{2}+Z_{2} / Z_{1}, Z_{1}=\rho_{s_{1}} v_{s_{1}}^{2} k_{1}$ and $Z_{2}=\rho_{s_{2}} v_{s_{2}}^{2} \kappa$. With the preceding results at hand, it is not difficult to prove that $t_{D_{l}}=\tau_{t_{l}}+\tau_{i_{l}}$ (fulfilling Eq. 25). Subindex $l$ denotes the limiting values. 
Arguments similar to those used to explain the electromagnetic HE are valid for our system. We can check in the resulting equations that the dwell time is proportional to the mechanical energy stored in the string, $t_{D_{l}}=H_{l} / j_{i n}$, with $j_{i n}=\rho_{s_{1}} \omega v_{s_{1}}^{2} k_{1}$. Therefore, the total group delay is proportional to the asymptotic mechanical energy, $\tau_{t_{l}}+\tau_{i_{l}}=$ $H_{l} / j_{\text {in }}$ and it saturates as the mechanical energy saturates and cannot be a transit time. We conclude the group delay in tunneling is not a transit time but a cavity lifetime.

\section{TUNNELING THROUGH AN ARRAY OF STRINGS}

Consider now a distributed Bragg reflector (DBR) made by stacking periodically $N$ times two taut strings $A_{1}$ and $A_{2}$ with different mass densities $\rho_{1}$ and $\rho_{2}$ and propagating velocities $v_{s_{1}}$ and $v_{s_{2}}$, respectively. The structure is sandwiched between two identical semi-infinite media $X$ and $Y$. For the sake of convenience, the constituent parameters of strings $X$ and $Y$ are equal to those of medium $A_{1}$. Figure 1 (c) shows a schematic representation of this system.

The amplitudes of the harmonic waves propagating through this system are computed with help of the transfer matrix method (TMM). ${ }^{47,48}$ Other quantities, like the transmission and reflection coefficients, the group delays, the stored energy, and the dwell time can be computed from these amplitudes using the formulas given in the previous section. Appendix D briefly reviews the TMM.

Fig. 3 compares the dispersion relation for an infinite array of bare taut strings with both the transmission coefficient $\mathcal{T}$ calculated for a finite DBR with $N=15$ periods and the corresponding transmission time $\tau_{t}$, c.f Figs. 3 (a), (b), and (c). Input parameters are given in the figure caption. A similar comparison is made in Figs. 3 (d), (e), and (f) for the case that string 1 is in air $\left(\mathcal{K}_{1}=0\right)$ and string 2 in an elastic foundation $\left(\mathcal{K}_{2} \neq 0\right)$. Thus, we can say the string 1 behaves like a well, while the string 2 acts a barrier for the incident wave.
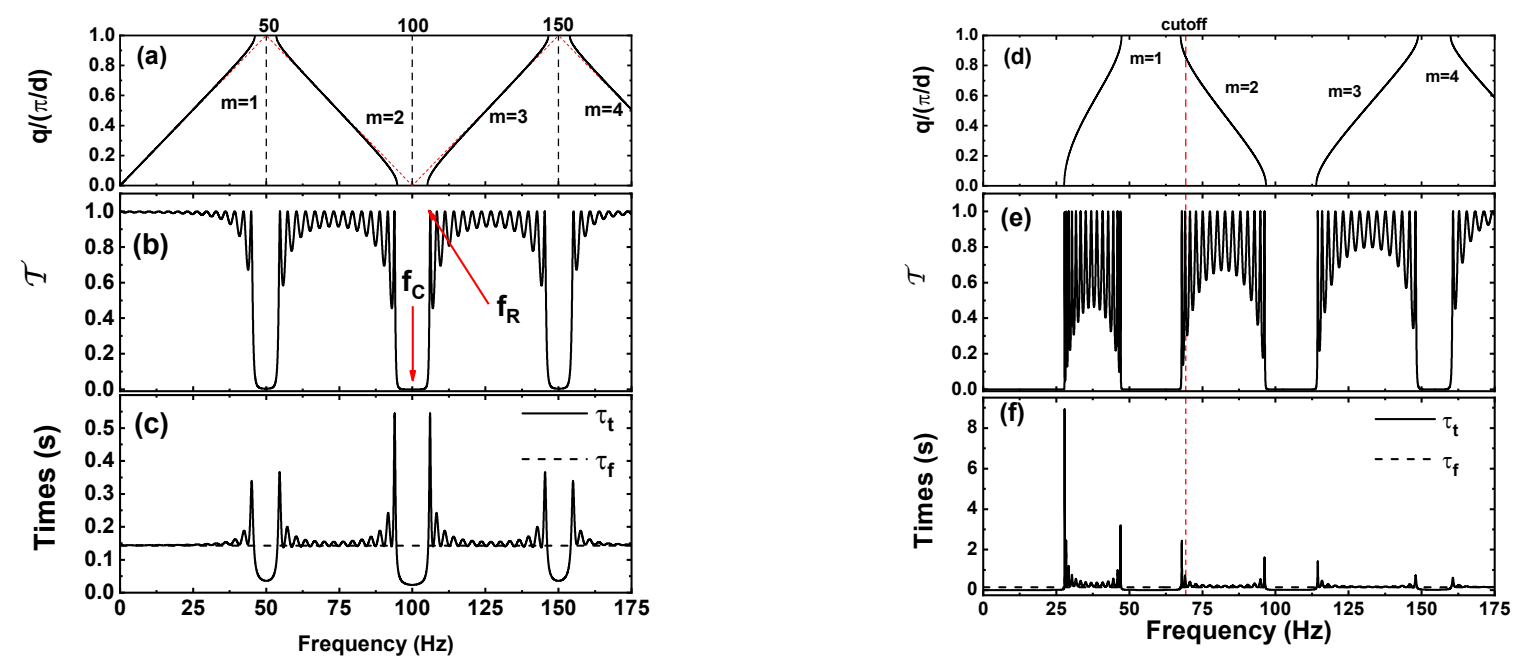

FIG. 3. (Color online). Bare taut string: (a) Exact (solid line) and approximate (dashed line) dispersion curves calculated as a function of the frequency (in $\mathrm{Hz}$ ) for an infinite array of bare strings. The length in the $q$ axis is normalized to unity. The central frequency of the stop bands are represented by vertical dashed lines. (b) Transmission coefficient vs. frequency for a finite DBR. (c) Transmission time $\tau_{t}$ (solid line) and free time $\tau_{f}$ (dashed line) (both in s) for a DBR. String on elastic foundation: (d) dispersion relation, (e) transmission coefficient, and (f) transmission time. The input parameters used for the string $A_{1}\left(A_{2}\right)$ are $\rho_{s_{1}}=0.52 \mathrm{~g} / \mathrm{cm}\left(\rho_{s_{2}}=1.0 \mathrm{~g} / \mathrm{cm}\right)$, and $v_{s_{1}}=40 \mathrm{~m} / \mathrm{s}\left(v_{s_{2}}=28.84 \mathrm{~m} / \mathrm{s}\right) ; d_{1}=10 \mathrm{~cm}$ and $d_{2}=21.63 \mathrm{~cm}$ are the width of the layers 1 and 2, respectively, $d=d_{1}+d_{2}$ is the period of the structure. The number of periods is assumed to be $N=15$ and $L_{t}=N d_{1}+(N-1) d_{2}$ is the total length of the structure. The elastic modulus of the foundations are $\mathcal{K}_{1}=0$ and $\mathcal{K}_{2}=9.87 \mathrm{kN} / \mathrm{m}^{2}$.

The transmission coefficient $\mathcal{T}$ is compared with the dispersion relation of an ideal periodic array in order to get physical insight. This analysis is valid for DBR with a large number of cells. In fact, the response of a DBR approaches thus of an infinite array for an increasing number of cells. ${ }^{9}$ For the limiting case of an infinity structure $(N \rightarrow \infty)$, the dispersion relation is cast into a simple analytic form (explicit calculations are given in Appendix D),

$$
\cos (q d)=\cos \left(k_{1} d_{1}+k_{2} d_{2}\right)-\frac{\epsilon^{2}}{2} \sin \left(k_{1} d_{1}\right) \sin \left(k_{2} d_{2}\right),
$$


where $\epsilon=\left(Z_{2}-Z_{1}\right) / \sqrt{Z_{1} Z_{2}}$. Only values of the wavenumber $q$ within the first $(-\pi / d<q<\pi / d)$ Brillouin zone (BZ) are going to be consider in what follows. In general, Eq. (33) must be solved numerically.

For the hypothetical case of having a single string in the unit cell of the array $\left(Z_{1}=Z_{2}\right.$ and $\left.\epsilon=0\right)$ the dispersion relation of the infinite string is folded into the first BZ producing a multiband structure. In this case, the dispersion relations is

$$
\pm q d=k_{1} d_{1}+k_{2} d_{2}+2 m \pi
$$

where $m \in \mathbb{Z}$ is the order that labels the different bands. Eq. (34) is obtained from (33) with $\epsilon=0$. When the two strings in the cell are different $\left(Z_{1} \neq Z_{2}\right.$ and $\left.\epsilon \neq 0\right)$, gaps are open at the center $q=0$ and the border $q= \pm \pi / d$ of the BZ. Gaps are also known as stop bands. Exact (solid line) and approximate (dashed line) dispersion curves are compared in Fig. 3(a).

The approximate expression (34) can be used to estimate the values of the frequency $f_{B_{m}}$ at the center of the stop bands. ${ }^{49}$ Substituting the linear dispersion relations of the bare taut strings, $k_{1}=\omega / v_{s_{1}}$ and $k_{2}=\omega / v_{s_{2}}$, into Eq. (34) we have

$$
f_{B_{m}}=\frac{m}{2}\left(\frac{d_{1}}{v_{s_{1}}}+\frac{d_{2}}{v_{s_{2}}}\right)^{-1}
$$

with $m=1,2, \ldots$ Even (odd) values of $m$ correspond to the gaps at the center (border) of the BZ. For the geometrical parameters used in Fig. 3, $f_{B_{m}}=(50 \mathrm{~Hz}) \mathrm{m}$. Numerical values are represented in Fig. 3(a) with vertical dashed lines.

The width of the first gap in the center of the BZ is maximum when the input parameters satisfy the relation $3 d_{1} / v_{s_{1}}=d_{2} / v_{s_{2}} .{ }^{6}$ The numerical parameters used in the calculations fulfill this relation. The gap widths in Fig. $3\left(\right.$ a) are $\Delta f_{2}=12.26 \mathrm{~Hz}$ and $\Delta f_{1}=\Delta f_{3}=9.86 \mathrm{~Hz}$ at center and border of the BZ, respectively.

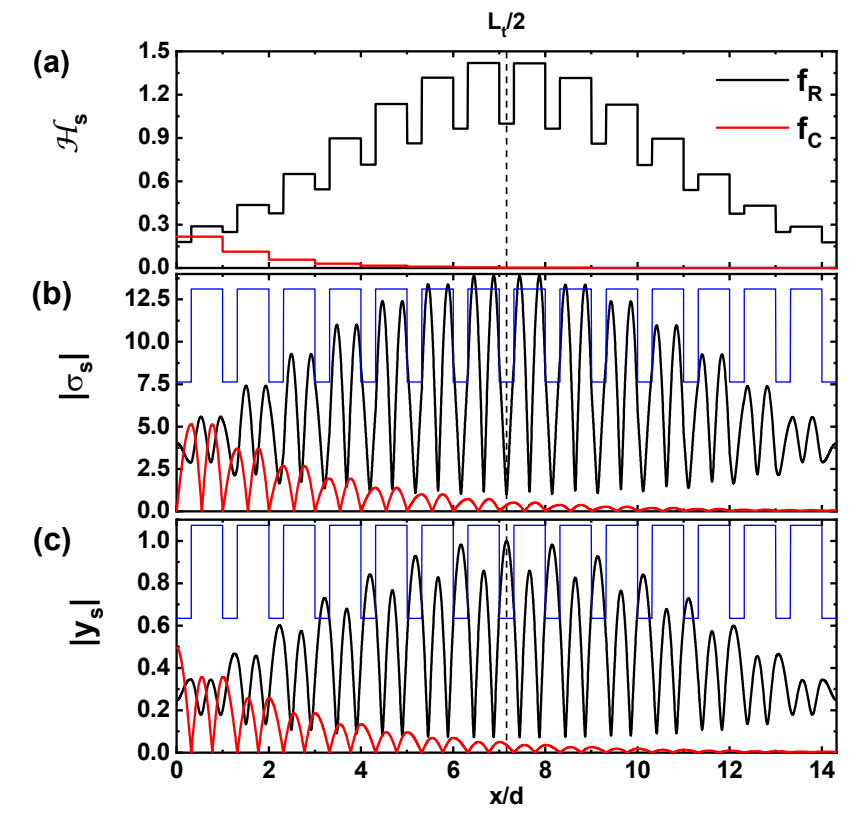

FIG. 4. (Color online) (a) Mechanical energy density $\mathcal{H}_{s}$ (normalized to $\mathcal{H}_{s}\left(f_{R}, L_{t} / 2\right)$ ), (b) stress $\left|\sigma_{s}\right|$ (normalized to $\left.\left|\sigma_{s}\left(f_{R}, L_{t} / 2\right)\right|\right)$, and (c) displacement field $\left|y_{s}\right|$ (normalized to $\left.\left|y_{s}\left(f_{R}, L_{t} / 2\right)\right|\right)$ in the DBR are plotted as a function of the normalized distance $x / d$ for the frequency values $f_{R}=107.5 \mathrm{~Hz}$ (black line) and $f_{C}=100 \mathrm{~Hz}$ (red line) of Fig. 3(b) . The impedance profile of the DBR is represented with a blue line. The vertical line is located at the center of the structure, $L_{t} / 2=2.62 \mathrm{~m}$.

Transmission bands and gaps of the finite DBR have practically the same width of those for the infinite array. The transmission bands have $N-1=14$ transmission peaks. While the response of the DBR of bare strings is characterized by transmission bands broader than the gaps, the elastic base in string 2 modifies dramatically the spectrum to have broader main gaps and deeper secondary gaps, c.f. Figs. 3(b) and (e). As for the single string, bands of Fig. 3(e) are shifted with respect to those of Fig. 3(b). However, in contrast with the single string, we find 
a transmission band below the cutoff frequency, $f_{c_{2}}=69 \mathrm{~Hz}$. The origin of this band is the tunneling of elastic energy trough the strings coupled to the elastic foundation. Such strings behave like potential barriers.

Transmission times $\tau_{t}$ for the bare string and the string on an elastic base are depicted in Figs. 3(c) and (f), respectively. $\tau_{t}$ oscillates around the free propagation time, $\tau_{f}=N d_{1} / v_{s_{1}}+(N-1) d_{2} / v_{s_{2}}$. We find that $\tau_{t}$ is larger than $\tau_{f}$ at the transmission resonances and lower than $\tau_{f}$ within the main transmission gaps. At the secondary gaps, however, $\tau_{t}=\tau_{f}$.

As for the single string, peaks and dips of both $\tau_{t}$ and $\mathcal{T}$ develop at the same spectral positions. With help of the TMM it is not difficult to prove that $\tau_{t}$ is proportional to $\mathcal{T}$ for a DBR with an arbitrary number of periods. This relation is immediate for coefficients of the last string and it is preserved for the remaining strings after successive multiplications by the transfer matrix. Therefore, we have that not only $\tau_{t}$, but also $H$ and $t_{D}$ are proportional to $\mathcal{T}$.

However, the prefactor of $\mathcal{T}$ in the general expression for $\tau_{t}$ substantially changes when the string is on an elastic foundation, as we have already noted for the single string. While, for the bare strings, $\tau_{t}$ is symmetric with respect to the central gap in the center of the BZ, $\tau_{t}$ decays with $f$ in the presence of the elastic foundation, c.f. Figs. 3(c) and (f).

In a symmetric environment (semi-infinite strings I and III are identical), transmission and reflection tunneling times are equal, $\tau_{t}=\tau_{r}$. This property is valid for any number of strings. It can be proved following procedures developed for electronic ${ }^{37}$ and phononic ${ }^{41}$ waves, which are based on the invariance of Eq. (2) under both time reversal and space reflection and the conservation of energy density flux.

The interference time (26) vanishes as long as there is no elastic base $\left(\omega_{c_{1}}=v_{s_{1}} \sqrt{\mathcal{K}_{1} / T}=0\right)$. Therefore, in accordance with Eq. (25), the dwell times is equal to the transmission time for bare strings, $t_{D}=\tau_{t}$. In order to illustrate why the life time of the wave increases when the transmission is enhanced, the mechanical energy density $\mathcal{H}_{s}$, the stress $\left|\sigma_{s}\right|$ and the displacement field $\left|y_{s}\right|$ are plotted as a function of the normalized distance $x / d$ in Fig. 4. These quantities are computed at the center of the gap (red line) and at a maximum intensity points next to the gap and located at its right side (black line). The points have spectral positions $f_{C}=100 \mathrm{~Hz}$ and $f_{R}=107.5 \mathrm{~Hz}$, as indicated in Fig. 3(b).

We find that the three quantities $\mathcal{H}_{s},\left|\sigma_{s}\right|$, and $\left|y_{s}\right|$ take a maximum value in the central strings where the string array is resonant at the frequency $f_{R}$ (for the mechanical energy is totally transmitted at these frequencies), while their intensities decrease with the distance off-resonance (for more of the energy is reflected back at $f_{C}$ ).

\section{CONCLUSIONS}

The tunneling of mechanical energy through taut strings have been studied. We have shown that a taut string on an elastic base can acts as a barrier where the impinging wave is reflected for a given frequency range, while the bare string behaves like a well. Therefore, string arrays with piecewise constitutive parameters can be built. This system is shown to be equivalent to heterostructures with alternating well and barrier layers studied in quantum mechanics or, more recently, in photonics and phononics. Similarly, in a set of $N$ identical multiple wells separated by finite barriers $N$-fold splitting of every energy level occurs. For very large $N$ one can speak about evolution of a discrete set of energy levels into energy bands in the taut string array.

Tunneling times such as the group delay, the dwell time and the interference time, as well as the relation between these quantities, have been defined for this system. We have discussed the main physical trends of the tunneling times in both single strings and arrays of strings with alternating constitutive parameters. In particular, the Hartman effect for infinite long barriers has been analyzed.

We hope that the present discussion motivate the experimental realization of the phenomena here described as well as further didactic and theoretical work.

\section{ACKNOWLEDGMENT}

D Villegas acknowledges the hospitality of Instituto de Física of Benemérita Universidad Autónoma de Puebla, México. 


\section{Appendix A: Derivation of the continuity equation for an elastic wave in a taut string on an elastic base}

The continuity equation for an elastic wave in a taut string connected to an elastic base is derived here. Results obtained here are also valid for the simpler case of a bare string. The starting point is the following set of equations

$$
\begin{aligned}
& y_{t}^{*} y_{t t}=v_{s}^{2} y_{t}^{*} y_{x x}-\omega_{c}^{2} y_{t}^{*} y, \\
& y_{t} y_{t t}^{*}=v_{s}^{2} y_{t} y_{x x}^{*}-\omega_{c}^{2} y_{t} y^{*},
\end{aligned}
$$

obtained by multiplying the wave equation (2) by $\partial_{t} y$ and $\partial_{t} y^{*}$, respectively. By adding the two equations and regrouping terms, we find

$$
\partial_{t}\left(y_{t}^{*} y_{t}\right)=v_{s}^{2}\left(y_{t}^{*} y_{x x}+u_{t} u y_{x x}^{*}\right)-\omega_{c}^{2} \partial_{t}\left(y^{*} y\right)
$$

The integral from $x_{1}$ to $x_{2}$ gives

$$
\partial_{t} \int_{x_{1}}^{x_{2}} d x\left(\left|y_{t}\right|^{2}+\omega_{c}^{2}|y|^{2}\right)=v_{s}^{2} \int_{x_{1}}^{x_{2}} d x\left(y_{t}^{*} y_{x x}+y_{t} y_{x x}^{*}\right) .
$$

Using a standard integration technique on the left side of Eq. (A3) and rearranging terms leads to

$$
\begin{aligned}
& \partial t \int_{x_{1}}^{x_{2}} d x\left(\frac{\rho_{s}}{2}\left|y_{t}\right|^{2}+\frac{\rho_{s}}{2} \omega_{c}^{2}|y|^{2}+\frac{\rho_{s}}{4} v_{s}^{2}\left(y_{x} y_{x}^{*}+y_{x}^{*} y_{x}\right)\right) \\
= & \left.\frac{\rho_{s}}{2} v_{s}^{2}\left(y_{t}^{*} y_{x}+y_{t} y_{x}^{*}\right)\right|_{x_{1}} ^{x_{2}},
\end{aligned}
$$

that can be simplified with help of the explicit expression of the stress function (4),

$$
\partial_{t} \int_{x_{1}}^{x_{2}} \mathcal{H}(x, t) d x=-\left(j\left(x_{2}, t\right)-j\left(x_{1}, t\right)\right),
$$

where

$$
\mathcal{H}(x, t)=\frac{\rho_{s}}{2}\left|y_{t}\right|^{2}+\frac{1}{4}\left(\sigma y_{x}^{*}+\sigma^{*} y_{x}\right)+\frac{\rho_{s}}{2} \omega_{c}^{2}|y|^{2}
$$

and

$$
j(x, t)=-\frac{\rho_{s}}{2}\left(\sigma^{*} y_{t}+\sigma y_{t}^{*}\right)
$$

Eq. (A5) can be further transformed by the mean-value theorem, ${ }^{50}$

$$
\frac{\partial}{\partial t} \mathcal{H}(\bar{x}, t)\left(x_{2}-x_{1}\right)=-\left(j\left(x_{2}, t\right)-j\left(x_{1}, t\right)\right)
$$

Therefore, after dividing by the integration interval $\Delta x=x_{2}-x_{1}$ and taking the limit $\Delta x \rightarrow 0$, we obtain

$$
\frac{\partial}{\partial t} \mathcal{H}(\bar{x}, t)+\frac{\partial}{\partial x} j(x, t)=0
$$

Since $\bar{x}$ is an arbitrary point of the interval $\left(x_{1}, x_{2}\right)$, Eq. (A9) has a general validity. This equation express the continuity equation for the energy density (11).

\section{Appendix B: Derivation of the relationship between the tunneling times}

In this section the relation between the tunneling times (25) is derived for an elastic wave in a taut string on an elastic base. Results obtained here are also valid for the simpler case of a bare string. The procedures we follow are similar to those used for quantum tunneling problems. ${ }^{42}$ In particular, we consider the sensitivity of the wave function to variations in frequency. ${ }^{35}$ 
We assume that a time-harmonic solution of the form $\tilde{y}(x, t)=y_{2}(x) e^{-i \omega t} x$ propagates in the region $0<0<L$. Substituting the energy density (14) into the equation for the mechanical energy (15) and using Eq. (4) yields

$$
H_{e f}=\frac{1}{2} \rho_{2}\left(\omega^{2}+\omega_{c_{2}}^{2}\right) \int_{0}^{L}\left|y_{2}\right|^{2} d x+\frac{1}{2} \rho_{2} v_{s_{2}}^{2} \int_{0}^{L}\left|\frac{d y_{2}}{d x}\right|^{2} d x .
$$

The second integral in Eq. (B1),

$$
I_{1}=\int_{0}^{L}\left|\frac{d y_{2}}{d x}\right|^{2} d x
$$

can be integrated by parts,

$$
I_{1}=\operatorname{Re}\left\{\left.y_{2} \frac{d y_{2}^{*}}{d x}\right|_{0} ^{L}-\int_{0}^{L} y_{2} \frac{d^{2} y_{2}^{*}}{d x^{2}} d x\right\}
$$

where the function $\operatorname{Re}(x)$ denote the real part of $x$. Notice that the displacement field $\tilde{y}(x, t)$ satisfies the Helmholtz equation,

$$
\frac{d^{2} y_{2}}{d x^{2}}+k_{2}^{2} y_{2}=0
$$

where $k_{2}^{2}=\left(\omega^{2}-\omega_{c_{2}}^{2}\right) / v_{s_{2}}^{2}$. By replacing the Helmholtz equation into Eq. (B3), one can obtain a new equation

$$
I_{1}=R e\left\{\left.y_{2} \frac{d y_{2}^{*}}{d x}\right|_{0} ^{L}+k_{2}^{2} \int_{0}^{L}\left|y_{2}\right|^{2} d x\right\} .
$$

Using Eq. (B5), the vibrational energy (B1) can be expressed as

$$
H_{e f}=\rho_{2} \omega^{2} \int_{0}^{L}\left|y_{2}\right|^{2} d x+\frac{1}{2} \rho_{2} v_{s_{2}}^{2} \operatorname{Re}\left\{\left.y_{2} \frac{d y_{2}^{*}}{d x}\right|_{0} ^{L}\right\} .
$$

The integral on the right-hand of Eq. (B6)

$$
I_{2}=\int_{0}^{L}\left|y_{2}\right|^{2} d x
$$

can be evaluated as

$$
I_{2}=\frac{v_{s_{2}}^{2}}{2 \omega} \operatorname{Re}\left\{\left.\left(\frac{\partial y_{2}}{\partial \omega} \frac{d y_{2}^{*}}{d x}-y_{2}^{*} \frac{\partial^{2} y_{2}}{\partial \omega \partial x}\right)\right|_{0} ^{L}\right\}
$$

(See Appendix C for further details). Therefore,

$$
H_{e f}=\frac{\rho_{2} v_{s_{2}}^{2} \omega}{2} \operatorname{Re}\left\{\left.\left(\frac{\partial y_{2}}{\partial \omega} \frac{d y_{2}^{*}}{d x}-y_{2}^{*} \frac{\partial^{2} y_{2}}{\partial \omega \partial x}\right)\right|_{0} ^{L}\right\}+\frac{\rho_{2} v_{s_{2}}^{2}}{2} \operatorname{Re}\left\{\left.y_{2} \frac{d y_{2}^{*}}{d x}\right|_{0} ^{L}\right\} .
$$

Inserting the boundary conditions (both continuity of displacement and stress fields in $x=0$ and $x=L$ ) in Eq. (B9), we obtain:

$$
\begin{aligned}
H_{e f} & =\frac{\omega}{2} \operatorname{Re}\left\{\left.\rho_{1} v_{s_{1}}^{2}\left(\frac{\partial y_{3}}{\partial \omega} \frac{d y_{3}^{*}}{d x}-y_{3}^{*} \frac{\partial^{2} y_{3}}{\partial \omega \partial x}\right)\right|_{x=L}\right\}-\frac{\omega}{2} \operatorname{Re}\left\{\left.\rho_{1} v_{s_{1}}^{2}\left(\frac{\partial y_{1}}{\partial \omega} \frac{d y_{1}^{*}}{d x}-y_{1}^{*} \frac{\partial^{2} y_{1}}{\partial \omega \partial x}\right)\right|_{x=0}\right\} \\
& +\frac{\rho_{1} v_{s_{1}}^{2}}{2} \operatorname{Re}\left\{\left.y_{3} \frac{d y_{3}^{*}}{d x}\right|_{x=L}\right\}+\frac{\rho_{1} v_{s_{1}}^{2}}{2} \operatorname{Re}\left\{\left.y_{1} \frac{d y_{1}^{*}}{d x}\right|_{x=0}\right\},
\end{aligned}
$$

Recalling how $y_{1}$ and $y_{2}$ are defined by Eq. (3), we finally have

$$
H_{e f}=\rho_{1} \omega v_{s_{1}}^{2} k_{1}|r|^{2} \frac{d \alpha_{r}}{d \omega}+\rho_{1} \omega v_{s_{1}}^{2} k_{1}|t|^{2} \frac{d \alpha_{t}}{d \omega}+\rho_{1} v_{s_{1}}\left(\omega \frac{d k_{1}}{d \omega}-k_{1}\right)|r| \sin \left(\alpha_{r}\right) .
$$

Normalizing the mechanical energy $H_{\text {ef }}$ to the incident flux $j_{i n}$, and using the proper definitions of tunneling times (20), (23) and (24), the identity (25) is obtained. 


\section{Appendix C: Calculation of the integral $I_{2}$}

The integral $I_{2}$ (B8) is computed in this section. We start from the following set of equations,

$$
\begin{gathered}
\frac{\partial y_{2}}{\partial \omega} \frac{d^{2} y_{2}^{*}}{d x^{2}}+k_{2}^{2} \frac{\partial y_{2}}{\partial \omega} y_{2}^{*}=0, \\
y_{2}^{*} \frac{\partial}{\partial \omega} \frac{d^{2} y_{2}}{d x^{2}}+y_{2}^{*} \frac{\partial}{\partial \omega}\left(k_{2}^{2} y_{2}\right)=0,
\end{gathered}
$$

which are obtained by multiplying the complex conjugate of the Helmholtz equation (B4) by $\partial y_{2} / \partial \omega$, and the derivative with respect to frequency of Helmholtz equation by $y_{2}^{*}$, respectively. Subtracting the two equations and rearranging terms, give us

$$
\left|y_{2}\right|^{2}=\frac{v_{s_{2}}^{2}}{2 \omega} \frac{\partial}{\partial x}\left(\frac{\partial y_{2}}{\partial \omega} \frac{\partial y_{2}^{*}}{\partial x}-y_{2}^{*} \frac{\partial^{2} y_{2}}{\partial \omega \partial x}\right) .
$$

Integrating over the interval $0 \leq x \leq L$, it reads,

$$
I_{2}=\int_{0}^{L}\left|y_{2}\right|^{2} d x=\frac{v_{s_{2}}^{2}}{2 \omega} \int_{0}^{L} \frac{\partial}{\partial x}\left(\frac{\partial y_{2}}{\partial \omega} \frac{\partial y_{2}^{*}}{\partial x}-y_{2}^{*} \frac{\partial^{2} y_{2}}{\partial \omega \partial x}\right) d x
$$

or, equivalently,

$$
I_{2}=\frac{v_{s_{2}}^{2}}{2 \omega} \operatorname{Re}\left\{\left.\left(\frac{\partial y_{2}}{\partial \omega} \frac{d y_{2}^{*}}{d x}-y_{2}^{*} \frac{\partial^{2} y_{2}}{\partial \omega \partial x}\right)\right|_{0} ^{L}\right\} .
$$

\section{Appendix D: Transfer matrix method}

Consider a finite number of taut strings sandwiched between two semi-infinite mediums. The transfer matrix method (TMM) is an efficient way of computing the amplitudes of the harmonic waves in each of the strings. ${ }^{47,48}$

The general form of the displacement field $y_{n, j}$ in the $j$ layer, with $j \in(1, \cdots, N)$, is equal to

$$
y_{n, j}(x, t)=\left(A_{n, j} e^{i k_{j} x}+B_{n, j} e^{-i k_{j} x}\right) e^{i \omega t},
$$

where $k_{j}$ and $d_{j}$ are the wave vector and thickness, respectively, of the $j$ layer with $0<x<d_{j}$. In the TMM, the displacement field is represented by a column vector of the amplitudes,

$$
C_{n, j}=\left(\begin{array}{c}
A_{n, j} \\
B_{n, j}
\end{array}\right) \text {. }
$$

In particular, the amplitudes of the transmitted and reflected displacement field are

$$
C_{Y}=\left(\begin{array}{c}
t \\
0
\end{array}\right), C_{X}=\left(\begin{array}{l}
1 \\
r
\end{array}\right),
$$

respectively. A relation between the amplitudes in consecutive layers is developed from the boundary conditions. This relation is written in form of a matrix. Multiplying $N$ times by this transfer matrix, the following relation is established between $C_{Y}$ and $C_{X}$,

$$
C_{X}=D_{X}^{-1} M_{1} \cdots M_{j} \cdots M_{N} D_{Y} C_{Y}=T C_{Y}
$$

where $M j=D_{j} P j D_{j}^{-1}$,

$$
\begin{aligned}
D_{j} & =\left(\begin{array}{cc}
1 & 1 \\
i \tilde{Z}_{j} & -i \tilde{Z}_{j}
\end{array}\right), \\
P_{j} & =\left(\begin{array}{cc}
e^{-i k_{j} d_{j}} & 0 \\
0 & e^{i k_{j} d_{j}}
\end{array}\right),
\end{aligned}
$$


$\tilde{Z}_{j}=\rho_{j} v_{s_{j}}^{2} k_{j}$ and $T=D_{X}^{-1} M_{1} \cdots M_{j} \cdots M_{N} D_{Y}$. Finally, $t$ and $r$ can be easily obtained from Eq. (D4).

Let us now consider a periodic structure with an infinite number of identical cells. The relation between the amplitudes of the displacement fields at two consecutive cells can be written either using the periodicity of the system or the transfer matrix,

$$
\left(\begin{array}{c}
A_{n+1,1} \\
B_{n+1,1}
\end{array}\right)=M\left(\begin{array}{c}
A_{n, 1} \\
B_{n, 1}
\end{array}\right)=e^{i q d}\left(\begin{array}{c}
A_{n, 1} \\
B_{n, 1}
\end{array}\right)
$$

where $M=D_{1}^{-1} D_{2} P_{2} D_{2}^{-1} D_{1} P_{1}$ is the transfer matrix, $q$ is the Bloch wave vector, and $d=d_{1}+d_{2}$ is the period of the structure. A nontrivial solutions of last equation can be achieved for

$$
\operatorname{Det}\left(M-e^{i q d} I\right)=0
$$

where $I$ is the identity matrix of order 2 . The dispersion relation (33) is the straightforward solution of this secular equation.

* diosdado@ifuap.buap.mx

$\dagger$ Corresponding author. e-mails: fdlp@unizar.es

1 S. He and J. D. Maynard, "Detailed measurements of inelastic scattering in Anderson localization," Phys. Rev. Lett. 57, 3171 (1986).

2 J. D. Maynard, "Colloquium: Acoustical analogs of condensed-matter problems," Rev. Mod. Phys. 73, 401-417 (2001).

3 N. D. Lanzillotti-Kimura, A. Fainstein, and B. Jusserand, "Phonon Bloch oscillations in acoustic-cavity structures," Phys. Rev. B 71, 041305(R) (2005).

${ }^{4}$ L. Gutiérrez, A. Díaz-de-Anda, J. Flores, R. A. Méndez-Sánchez, G. Monsivais, and A. Morales, "Wannier-Stark Ladders in One-Dimensional Elastic Systems," Phys. Rev. Lett. 97, 114301 (2006).

${ }^{5}$ G. Monsivais, R. A. Méndez-Sánchez, A. Díaz-de-Anda, J. Flores, L. Gutiérrez, and A. Morales, "Elastic Wannier-Stark ladders in torsional waves," J. of Mechanics of Materials and Structures 2, 1629-1637 (2007).

${ }^{6}$ N. D. Lanzillotti-Kimura, A. Fainstein, C. A. Balseiro, and B. Jusserand, "Phonon engineering with acoustic nanocavities: Theoretical considerations on phonon molecules, band structures, and acoustic Bloch oscillations," Phys. Rev. B 75, 024301 (2007).

7 A. Morales, A. Díaz-de-Anda, J. Flores, L. Gutiérrez, R. A. Méndez-Sánchez, G. Monsivais, and P. Mora, "Doorway states in quasione-dimensional elastic systems," EPL 99, 54002 (2012).

8 F. de León-Pérez and R. Pérez-Álvarez, "Long-wavelength nonpolar optical modes in semiconductor heterostructures: Continuum phenomenological model," Phys. Rev. B 61, 4820 (2000).

${ }^{9}$ F. de León-Pérez and R. Pérez-Álvarez, "Phonon propagation in nonpolar semiconductor heterostructures," Phys. Rev. B 63, 245304 (2001).

10 M. Sigalas, M. S. Kushwaha, E. N. Economou, M. Kafesaki, I. E. Psarobas, and W. Steurer, "Classical vibrational modes in phononic lattices: theory and experiment," Z. Kristallogr. 220, 765 (2005).

11 T. E. Hartman, "Tunneling of a wave packet," J. Appl. Phys. 33, 3427-3433 (1962).

12 A. Enders and G. Nimtz, "Evanescent-mode propagation and quantum tunneling," Phys. Rev. E 48, 632 (1993).

13 A. Enders and G. Nimtz, "Zero-time tunneling of evanescent mode packets," J. Phys. I France 3, 1089 (1993).

14 A. M. Steinberg, P. G. Kwiat, and R. Y. Chiao, "Measurement of the single-photon tunneling time," Phys. Rev. Lett. 71, 708 (1993).

15 Ch. Spielmann, R. Szipocs, A. Stingl, and F. Krausz, "Tunneling of optical pulses through photonic band gaps," Phys. Rev. Lett. 73, 2308 (1994).

16 S. Longhi, M. Marano, P. Laporta, and M. Belmonte, "Superluminal optical pulse propagation at $1.5 \mu \mathrm{m}$ in periodic fiber Bragg gratings," Phys. Rev. E 64, 055602(R) (2001).

17 Ph. Balcou and L. Dutriaux, "Time delay of scattering processes," Phys. Rev. Lett. 78, 851 (1997).

18 A. Hache and L. Poirier, "Long-range superluminal pulse propagation in a coaxial photonic crystal," Appl. Phys. Lett. 80, 518 (2002).

19 H. G. Winful, "Tunneling time, the Hartman effect, and superluminality: A proposed resolution of an old paradox," Phys. Reports 436, 1-69 (2006).

${ }^{20}$ D. Villegas, F. de León-Pérez, and R. Pérez-Álvarez, "Tunneling time for phonons: dependence on the systems size," Phys Status Solidi (b) 242, 1767 (2005).

21 A. Huynh, N. Lanzillotti-Kimura, B. Jusserand, B. Perrin, A. Fainstein, M. Pascual-Winter, E. Peronne, and A. Lemaître, "Subterahertz Phonon Dynamics in Acoustic Nanocavities," Phys. Rev. Lett. 97, 115502 (2006).

${ }^{22}$ D. Villegas, F. de León-Pérez, R. Pérez-Álvarez, and J. Arriaga, "Phonon tunneling through a double barrier system," Physica B 463, 7 (2015).

23 J. L. Rose, Ultrasonic guided wave in solid media (Cambridge University Press, 2014). 
${ }^{24}$ K. F. Graff, Wave motion in elastic solids (Dover Publications, Inc, New York 1991).

${ }^{25}$ U. Hassan, Z. Usman, and M. S. Anwar, "Video-based spatial portraits of a nonlinear vibrating string," Am. J. Phys. 80, 862-869 (2012).

26 D. R. Rowland, "The surprising influence of longitudinal motion in vibrating strings: Comment on Video-based spatial portraits of a nonlinear vibrating string [Am. J. Phys. 80 (10), 862-869 (2012)]," Am. J. Phys. 83, 1058-1060 (2015).

27 S. B. Whitfield and K. B. Flesch, "An experimental analysis of a vibrating guitar string using high-speed photography," Am. J. Phys. 82, 102-109 (2014).

28 D. R. Rowland, "Understanding nonlinear effects on wave shapes: Comment on An experimental analysis of a vibrating guitar string using high-speed photography [Am. J. Phys. 82 (2),102-109 (2014)]," Am. J. Phys. 83, 979-983 (2015).

29 D. R. Rowland and C. Pask, "The missing wave momentum mystery," Am. J. Phys. 67, 378-388 (1999).

30 D. J. Griffiths and C. A. Steinke, "Waves in locally periodic media," Am. J. Phys. 69, 137-154 (2001).

31 D. R. Rowland, "Parametric resonance and nonlinear string vibrations," Am. J. Phys. 72 (6), $758-766$ (2004).

32 E. I. Butikov, "Peculiarities in the energy transfer by waves on strained strings," Phys. Scr. 88, 065402 (2013).

33 G. M. Gehring, A. C. Liapis, and R. W. Boyd, "Tunneling delays in frustrated total internal reflection," Phys. Rev. A 85, $032122(2012)$.

34 S. Mizuno, and S. Tamura, "Theory of acoustic-phonon transmission in finite-size superlattice systems," Phys. Rev. B 45, 734 (1992).

35 F.T. Smith, "Lifetime matrix in collision theory," Phys. Rev. 118, 349 (1960).

36 B. A van Tiggelent, A. Tipt and A. Lagendijktt, "Dwell times for light and electrons," J. Phys. A Math. Gen. 26, 1731 (1993).

37 E. H. Hauge, J. P. Falck, and T. A. Fjeldly, "Transmission and refletion times for scattering of wave packets off tunneling barriers," Phys. Rev. B 36, 4203 (1987).

38 D. Villegas, F. de León-Pérez, and R. Pérez-Álvarez, "Tunneling time of long-wavelength phonons through semiconductor heterostructures," Phys. Rev. B 71, 035322 (2005).

39 H.G. Winful, "Nature of "Superluminal" Barrier Tunneling," Phys. Rev. Lett. 90, 023901 (2003).

40 D. Villegas, J. Arriaga, F. de León-Pérez, and R. Pérez-Álvarez, "Goos-Hänchen effect for optical vibrational modes in a semiconductor structure," J. Phys.: Condens. Matter 29, 125301 (2017).

41 Z. Lazcano, P. L. V. Negrín, D. Villegas, J. Arriaga and R. Pérez-Álvarez, "Tunneling times of acoustic phonon packets through a distributed Bragg reflector," Nanoscale Research Lett. 9, 44 (2014).

42 H. G. Winful, "Delay Time and the Hartman Effect in Quantum Tunneling," Phys. Rev. Lett. 91, 260401 (2003).

43 Z. Wu, Kai Chang, J. T. Liu, X. J. Li, and K. S. Chan, "The Hartman effect in graphene," J. of Appl. Phys. 105, 043702 (2009).

44 S. Dutta Gupta R. Arun, and G. S. Agarwal, "Subluminal to superluminal propagation in a left-handed medium," Phys. Rev. B 69, 113104 (2004).

${ }^{45}$ H.G. Winful, and C. Zhang, "Tunneling delay time in frustrated total internal reflection," Phys. Rev. A 79, 023826 (2009).

46 J. V. Radovanovic, I. Ilic, P. P. Belicev, L. Hadzievski, and V. B. Milanovica, "Tunneling times in dispersive and third-order nonlinear optical metamaterials," J. of Nanophotonics 5, 051802-1 (2011).

47 P. Yeh, A. Yariv, and Chi-Shain Hong, "Electromagnetic propagation in periodic stratified media. I. General theory," J. Opt. Soc. Am. 67, 423 (1977).

${ }^{48}$ P. Yeh, and A. Yariv, Optical Waves in Crystals (John Wiley Sons, New York, 1984).

49 G. N. Aliev, B. Goller, D. Kovalev, and P. A. Snow, "Hypersonic acoustic mirrors andmicrocavities in porous silicon," Appl. Phys. Lett. 96, 124101 (2010).

50 T. M. Apostol, Mathematical analysis (Addison-Wesley Publishing Company, United States of America Fifth printing, 1981). 